\title{
Angelic Semantics of Fine-Grained Concurrency ${ }^{\star}$
}

\author{
Dan R. Ghica and Andrzej S. Murawski \\ Oxford University Computing Laboratory \\ Wolfson Building, Parks Road, Oxford OX1 3QD, UK \\ \{Dan.Ghica, Andrzej. Murawski\}@comlab.ox.ac.uk
}

\begin{abstract}
We introduce a game model for a procedural programming language extended with primitives for parallel composition and synchronization on binary semaphores. The model uses an interleaved version of Hyland-Ong-style games, where most of the original combinatorial constraints on positions are replaced with a simple principle naturally related to static process creation. The model is fully abstract for mayequivalence.
\end{abstract}

\section{Introduction}

The two major paradigms of concurrent programming are message-passing and shared-variable. The latter style of programming is closer to the underlying machine model, which makes it both more popular and more "low-level" (and more error-prone) than the former. This constitutes very good motivation for the study of such languages. Concurrent shared-variable programming languages themselves can come in several varieties:

- Fine-grained languages have designated atomic actions which are implemented directly by the hardware on which the program is executed. In contrast, coarse-grained programming languages can specify sequences of actions to appear as indivisible.

- Languages with static process creation execute statements in parallel and then synchronize on the completion of all the statements. Conversely, $d y$ namic process creation languages can create wholly autonomous new threads of execution.

- The procedure invocation mechanism can be call-by-name or call-by-value.

Any combination of the features above is possible and yields interesting programming languages. In this paper we consider fine-grained, static, call-by-name languages. We found that this particular set of choices is most naturally suited to the particular semantic model we intend to present.

Our language comes very close to Brookes's Parallel Algol (PA) [1, which is a coarse-grained, static, call-by-name language. Whereas PA uses a coarse-grained await construct, we use fine-grained semaphores, with atomic operations grab

\footnotetext{
* Work funded by British EPSRC, Canadian NSERC and St John's College, Oxford.
} 
and release. Additionally, unlike PA, our language allows side-effects in expressions. But otherwise it is very similar to PA, and both are quite faithful to Reynolds's principles of combining call-by-name $\lambda$-calculus with local-variable imperative programming.

For sequential Algol, the combination of procedures and state gives rise to difficult semantic problems [2], which were first given an adequate solution relatively recently by Abramsky and McCusker using game semantics [3]. Their game model of Algol uses Hyland-Ong-style (HO) games which had previously been used to model sequential functional computation, notably for the language PCF [4]. Since game models are strikingly concurrent, adapting them to the analysis of parallel computation is a natural step. As it will be seen in this paper, the game model of concurrency is substantially simpler than that of sequentiality. One can think of sequentiality as a highly-constrained and deterministic form of interleaving of concurrent actions, this being reflected by the nature of the rules governing the HO games. To model concurrency we renounce almost all the HO rules, including the most basic one, the embodiment of sequentiality, alternation, and replace them with a single principle that is an immediate reflection on the nature of static concurrency. The relative simplicity of our model is best illustrated by the direct definability proof. While the factorization method seems possible in principle, it would perhaps obscure the connection between the concurrent nature of computation and the concurrent nature of games. The resultant game model is fully abstract with respect to may-equivalence. Therefore it can be used to reason about safety properties, but not liveness (deadlock-freeness).

Concurrent games, using a true concurrency representation, have been used by Abramsky and Melliès to model multiplicative additive linear logic [5]. Abramsky also made the first attempt to model PA using resumption-style games [6], but the theoretical properties of that model have not been investigated. We found that the interleaved representation is the most suitable for our language, because it deals more easily with the possibility of synchronization which happens either inherently at process creation and termination, or explicitly through the usage of semaphores.

Laird's game model of synchronous message-passing concurrency [7] is the work most closely related to ours. It draws from the $\mathrm{HO}$ model, and it also uses a non-alternating interleaved representation of concurrency. However, the technical differences are substantial. Laird's model introduces additional structure (concurrency pointers, to explicitly model threads) and additional conditions (pointer-blindness, to cut-down the model) in order to set up a framework compatible with the PCF constraints (visibility, innocence, well-bracketing). By contrast, our approach is more direct and yields an explicit model which seems more accessible.

Other work on denotational models for shared-variable concurrency we consider related to ours are Brookes's full abstraction result for a transition-trace model of a ground-type programming language [8] and his relational parametric model of PA [1]. Also interesting is Röckl and Sangiorgi's process semantics of PA 
using the $\pi$-calculus [9]. A representation of our game model into the $\pi$-calculus seems possible, which would give a fully abstract $\pi$-calculus model of PA.

\section{Syntax and Operational Semantics}

The types are $\beta:=\exp \mid \operatorname{com}$ and $\theta::=\beta \mid$ var $|\operatorname{sem}| \theta \rightarrow \theta$. The type judgements are of form $\Gamma \vdash M: \theta$ where $\Gamma$ maps identifiers to types. The typing rules are those of Idealized Algol (IA) with active expressions plus rules for the following new terms:

$$
\begin{array}{ccc}
\frac{\Gamma \vdash C_{1}: \operatorname{com} \quad \Gamma \vdash C_{2}: \operatorname{com}}{\Gamma \vdash C_{1} \| C_{2}: \operatorname{com}} & & \frac{\Gamma, x: \operatorname{sem} \vdash M: \beta}{\Gamma \vdash \operatorname{newsem} x:=n \operatorname{in} M: \beta} \\
\frac{\Gamma \vdash S: \operatorname{sem}}{\Gamma \vdash \operatorname{grab}(S): \operatorname{com}} & \frac{\Gamma \vdash S: \operatorname{sem}}{\Gamma \vdash \operatorname{release}(S): \operatorname{com}}
\end{array}
$$

We define the semantics of the language using a (small-step) transition relation $\Sigma \vdash M, s \longrightarrow M^{\prime}, s^{\prime} . \Sigma$ is a set of names of variables denoting memory cells and of semaphores denoting locks; $s, s^{\prime}$ are states, i.e. functions $s, s^{\prime}: \Sigma \rightarrow \mathbb{N}$, and $M, M^{\prime}$ are terms.

The reduction rules specific to our language are those for parallel composition, semaphore manipulation and binding and we give them below.

$$
\begin{gathered}
\frac{\Sigma \vdash C_{1}, s \longrightarrow C_{1}^{\prime}, s^{\prime}}{\Sigma \vdash C_{1}\left\|C_{2}, s \longrightarrow C_{1}^{\prime}\right\| C_{2}, s^{\prime}} \quad \frac{\Sigma \vdash C_{2}, s \longrightarrow C_{2}^{\prime}, s^{\prime}}{\Sigma \vdash C_{1}\left\|C_{2}, s \longrightarrow C_{1}\right\| C_{2}^{\prime}, s^{\prime}} \\
\frac{\Sigma, v \vdash C[v / x], s \otimes(v \mapsto n) \longrightarrow C^{\prime}, s^{\prime} \otimes\left(v \mapsto n^{\prime}\right)}{\Sigma \vdash \operatorname{newsem} x:=n \operatorname{in} C, s \longrightarrow \operatorname{newsem} x:=n^{\prime} \operatorname{in} C^{\prime}[x / v], s^{\prime}} \\
\Sigma \vdash \operatorname{grab}(v), s \otimes(v \mapsto 0) \longrightarrow \operatorname{skip}, s \otimes(v \mapsto 1) \\
\Sigma \vdash \operatorname{release}(v), s \otimes(v \mapsto n) \longrightarrow \operatorname{skip}, s \otimes(v \mapsto 0) \quad n>0
\end{gathered}
$$

Semaphores are interpreted in a standard way, using stateful locks, $v$. If $v$ is 0 then the semaphore can be grabbed, which changes its state to 1 ; if $v$ is non-zero then the semaphore can be released, which changes its state back to 0 . Note that semaphore operations are atomic, i.e. they cannot be interrupted by other concurrent processes.

It is common to identify var with $(\exp \rightarrow \mathbf{c o m}) \times \exp$ and use a variable constructor mkvar : $(\exp \rightarrow \mathbf{c o m}) \rightarrow \exp \rightarrow \operatorname{var}[2]$. In the same "objectoriented" spirit, we identify sem with $\operatorname{com} \times \mathbf{c o m}$ and introduce a semaphore constructor mksem : com $\rightarrow$ com $\rightarrow$ sem.

We use the following abbreviations: $M, s \Downarrow$ if $\exists s^{\prime}, M, s \longrightarrow^{*} c, s^{\prime}$, with $c \in \mathbb{N} \cup$ \{skip\}, and $M \Downarrow$ if $M$ is closed and $M, \emptyset \Downarrow$. We define a contextual approximation relation $\Gamma \vdash M_{1} \check{\sim}_{\theta} M_{2}$ by $\forall \mathcal{C}[-]:$ com, $\mathcal{C}\left[M_{1}\right] \Downarrow$ implies $\mathcal{C}\left[M_{2}\right] \Downarrow$, where $\mathcal{C}\left[M_{i}\right]$ are closed programs of com type. Contextual equivalence $\left(\Gamma \vdash M_{1} \cong{ }_{\theta} M_{2}\right)$ is defined as $\Gamma \vdash M_{1} \check{\sim}_{\theta} M_{2}$ and $\Gamma \vdash M_{2} \check{\sim}_{\theta} M_{1}$.

Note that the definition of termination $M \Downarrow$ is angelic. We consider a term to terminate if there exists a terminating evaluation. However, the evaluation is 
not deterministic, so it is possible that a term has both terminating and nonterminating evaluations. Moreover, we do not differentiate between the various reasons that termination might fail. In our language this can happen either because of infinite reductions (divergence, e.g. fix $(\lambda x . x)$ ) or stuck configurations (deadlock, e.g. newsem $s:=0$ in $\operatorname{grab}(s) ; \operatorname{grab}(s)$ ).

\section{Game Semantics}

Game semantics models computation as a game between a Proponent (P), representing a term, and an Opponent $(\mathrm{O})$, representing the environment of the term. Any play of the game is an interaction consisting of basic actions called moves, which are of two kinds: questions and answers. The fundamental rule is that questions can only be asked if they are justified by some previous question, and answers can be given only to relevant questions. A common metaphor is that of polite conversation: one must not ask irrelevant questions or provide unrequested answers. In addition, any play must obey other various rules, which are particular and intimately related to the kind of computations one is interested in modeling. $\mathrm{P}$ must always play according to a strategy that interprets the term. O does not play using some pre-determined strategy, but it still needs to behave according to the rules of play.

The game-semantic approach, which is highly intensional and interactive, is particularly well suited for modeling concurrent programming languages. Ironically perhaps, the greatest initial success of game semantics was in providing models for sequential computation. Sequentiality is a straitjacketed form of interaction, and its game models reflect this situation by being governed by a number of combinatorial rules.

The essential rule common to all sequential games, is that of alternation: $\mathrm{O}$ and $\mathrm{P}$ must take turns. In order to model concurrency we also discard this rule. The "static" style of concurrency of our programming language requires that any process starting sub-processes must wait for the children to terminate in order to terminate itself. At the level of games, this is reflected by the following principle:

In any prefix of a play, if a question is answered then that question and all questions justified by it are answered exactly once.

It is helpful to spell out this property using two simpler and more precise rules:

Forking Only a question that has not been answered can be used as a justifier for future moves.

Waiting A question can be answered only after all the questions justified by it have been answered.

A lot of by now standard definitions in game semantics can be adapted to the new setting. We detail the similarities and differences in what follows. 


\subsection{Arenas}

The definition of arenas remains standard. An arena $A$ is a triple $\left\langle M_{A}, \lambda_{A}, \vdash_{A}\right\rangle$ where $M_{A}$ is a set of moves, $\lambda_{A}: M_{A} \rightarrow\{O, P\} \times\{Q, A\}$ is a function determining for each $m \in M_{A}$ whether it is an Opponent or a Proponent move, and a question or an answer. We write $\lambda_{A}^{O P}, \lambda_{A}^{Q A}$ for the composite of $\lambda_{A}$ with respectively the first and second projections. $\vdash_{A}$ is a binary relation on $M_{A}$, called enabling, satisfying

- if $m \vdash_{A} n$ for no $m$ then $\lambda_{A}(n)=(O, Q)$,

- if $m \vdash_{A} n$ then $\lambda_{A}^{O P}(m) \neq \lambda_{A}^{O P}(n)$,

- if $m \vdash_{A} n$ then $\lambda_{A}^{Q A}(m)=Q$.

If $m \vdash_{A} n$ we say that $m$ enables $n$. We shall write $I_{A}$ for the set of all moves of $A$ which have no enabler; such moves are called initial. Note that an initial move must be an Opponent question.

The product $(A \times B)$ and arrow $(A \Rightarrow B)$ arenas are defined by:

$$
\begin{aligned}
M_{A \times B} & =M_{A}+M_{B} & M_{A \Rightarrow B} & =M_{A}+M_{B} \\
\lambda_{A \times B} & =\left[\lambda_{A}, \lambda_{B}\right] & \lambda_{A \Rightarrow B} & =\left[\left\langle\lambda_{A}^{P O}, \lambda_{A}^{Q A}\right\rangle, \lambda_{B}\right] \\
\vdash_{A \times B} & =\vdash_{A}+\vdash_{B} & \vdash_{A \Rightarrow B} & =\vdash_{A}+\vdash_{B}+\left\{(b, a) \mid b \in I_{B} \text { and } a \in I_{A}\right\}
\end{aligned}
$$

where $\lambda_{A}^{P O}(m)=O$ if and only if $\lambda_{A}^{O P}(m)=P$.

An arena is called flat if its questions are all initial (consequently the P-moves can only be answers). The arenas used to interpret base types are all flat:

\begin{tabular}{c|c|c|c|c|c} 
Arena & O-question & P-answers & Arena & O-question & P-answers \\
\hline \hline$\llbracket \mathbf{c o m} \rrbracket$ & run & $o k$ & $\llbracket \exp \rrbracket$ & $q$ & $n$ \\
\hline$\llbracket \mathbf{v a r} \rrbracket$ & read & $n$ & $\llbracket \mathbf{s e m} \rrbracket$ & $\begin{array}{c}\text { grab } \\
\text { release }\end{array}$ & $\begin{array}{c}\text { ok } \\
\text { write }(n)\end{array}$ \\
& ok
\end{tabular}

Note that $\llbracket \mathbf{s e m} \rrbracket$ is isomorphic to $\llbracket \mathbf{c o m} \rrbracket \times \llbracket \mathbf{c o m} \rrbracket$ and $\llbracket \mathbf{v a r} \rrbracket=\llbracket \mathbf{c o m} \rrbracket^{\omega} \times \llbracket \mathbf{e x p} \rrbracket$, where by $\llbracket \mathbf{c o m} \rrbracket^{\omega}$ we mean the product of countably many copies of $\llbracket \mathbf{c o m} \rrbracket$.

\subsection{Positions}

A justified sequence in arena $A$ is a finite sequence of moves of $A$ equipped with pointers. The first move is initial and has no pointer, but each subsequent move $n$ must have a unique pointer to an earlier occurrence of a move $m$ such that $m \vdash_{A} n$. We say that $n$ is (explicitly) justified by $m$ or, when $n$ is an answer, that $n$ answers $m$. Note that interleavings of several justified sequences may not be justified sequences; instead we shall call them shuffled sequences.

If a question does not have an answer in a justified sequence, we say that it is pending in that sequence. In what follows we use the letters $q$ and $a$ to refer to question- and answer-moves respectively, $m$ will be used for arbitrary moves and $m_{A}$ will be a move from $M_{A}$. When we write justified sequences we only indicate those justification pointers which cannot be inferred without ambiguity from the structure of the sequence.

Next we define what sequences of moves are considered "legal": 
Definition 1. The set $P_{A}$ of positions (or plays) over $A$ consists of the justified sequences s over $A$ which satisfy the two conditions below.

FORK : In any prefix $s^{\prime}=\cdots q^{\aleph} \cdots m$ of $s$, the question $q$ must be pending before $m$ is played.

WAIT : In any prefix $s^{\prime}=\cdots q^{\aleph} \ldots a$ of $s$, all questions justified by $q$ must be answered.

The simplest sequences of moves that violate FORK and WAIT respectively are:

$$
q \leftarrow a \quad \text { and } \quad q \leftarrow_{q} a
$$

The notion of a play is stable with respect to various swapping operations:

Lemma 1. - If $s m_{1} m_{2} \in P_{A}$ and $\lambda_{A}^{O P}\left(m_{1}\right)=\lambda_{A}^{O P}\left(m_{2}\right)$, then $s m_{2} m_{1} \in P_{A}$.

- If smq $\in P_{A}$ and $q$ is not justified by $m$ then $s q m \in P_{A}$.

- If sqa $\in P_{A}$ and $a$ is not justified by $q$, then saq $\in P_{A}$.

- If $s a_{1} a_{2} \in P_{A}$ and $s a_{2} a_{1}$ satisfies WAIT then $s a_{2} a_{1} \in P_{A}$.

Note that the definitions of $A \times B$ and $A \Rightarrow B$ no longer imply the usual switching condition, which characterizes sequential execution.

Definition 2. A play $s \in P_{A}$ is complete iff no questions in $s$ are pending.

The following notations will be useful. For two shuffled sequences $s_{1}$ and $s_{2}$, $s_{1} \amalg s_{2}$ will denote the set of all interleavings of $s_{1}$ and $s_{2}$. For two sets of shuffled sequences $S_{1}$ and $S_{2}: S_{1} \amalg S_{2}=\bigcup_{s_{1} \in S_{1}, s_{2} \in S_{2}} s_{1} \amalg s_{2}$. Given a set $X$ of shuffled sequences, we define $X^{0}=X, X^{i+1}=X^{i} \amalg X$. Then $X^{\circledast}$, called iterated shuffle of $X$, is defined to be $\bigcup_{i \in \mathbb{N}} X^{i}$.

\subsection{Strategies}

Strategies describe the way programs (represented by $\mathrm{P}$ ) interact with their environment (represented by $\mathrm{O}$ ).

Definition 3. A strategy $\sigma$ on $A$ (written $\sigma: A$ ) is a prefix-closed subset of $P_{A}$, which is O-complete, i.e. if $s \in \sigma$ and $s o \in P_{A}$, where $o$ is an (occurrence of an) O-move, then so $\sigma$.

O-completeness signifies the fact that the environment cannot be controlled during the interaction, and can make any legal move at any time. We will often define strategies using sets of sequences omitting the prefix- or O-closure. We will say that $\mathrm{P}$ has a response at position $s$ (when following $\sigma$ ) if $s p \in \sigma$ for some Pmove $s$. The set of non-empty complete plays of a strategy $\sigma$ will be denoted by $\operatorname{comp}(\sigma)$.

Two strategies $\sigma: A \Rightarrow B$ and $\tau: B \Rightarrow C$ can be composed by considering their possible interactions in the shared arena $B$. Moves in $B$ are subsequently hidden yielding a sequence of moves in $A$ and $C$. 
Each play in $A \Rightarrow B$ has a unique initial move, but plays in $\tau$ may use several initial $B$-moves. The latter corresponds to multiple uses of the argument of type $B$. Thus, when the strategies are interacting, positions of $\sigma$ will be replicated in order to allow for any number of copies of $\sigma$ to be "used" by $\tau$.

More formally, let $u$ be a sequence of moves from arenas $A, B$ and $C$ with justification pointers from all moves except those initial in $C$ such that pointers from moves in $C$ cannot point to moves in $A$ and vice versa. Define $u \uparrow B, C$ to be the subsequence of $u$ consisting of all moves from $B$ and $C$ (pointers between $A$-moves and $B$-moves are ignored). $u \uparrow A, B$ is defined analogously (pointers between $B$ and $C$ are then ignored). We say that $u$ is an interaction sequence of $A, B$ and $C$ if $u \uparrow A, B \in P_{A \Rightarrow B}^{\circledast}$ and $u \uparrow B, C \in P_{B \Rightarrow C}$. The set of all such sequences is written as $\operatorname{int}(A, B, C)$. Then the interaction sequence $\sigma$ 々 $\tau$ of $\sigma$ and $\tau$ is defined by $\sigma \xi \tau=\left\{u \in \operatorname{int}(A, B, C) \mid u \uparrow A, B \in \sigma^{\circledast}, u \uparrow B, C \in \tau\right\}$.

Suppose $u \in \operatorname{int}(A, B, C)$. Define $u \uparrow A, C$ to be the subsequence of $u$ consisting of all moves from $A$ and $C$, but where there was a pointer from a move $m_{A} \in M_{A}$ to an initial move $m_{B} \in M_{B}$ extend the pointer to the initial move in $C$ which was pointed to from $m_{B}$. Then the composite strategy $\sigma ; \tau$ is defined to be $\{u \uparrow A, C \mid u \in \sigma 々 \tau\}$.

\subsection{Saturated Strategies}

The original definition of strategies is inherently sequential. It relies on sequences of moves. Clearly, this cannot be sufficient to interpret concurrent computation. Sequences of events represent only one of possibly many observations of events which occur in parallel. Much of the ordering of the events present in such a sequence is arbitrary. We must consider strategies containing all possible such (sequential) observations of (parallel) interactions. In other words, strategies must be closed under inessential (i.e. unobservable) differences in the order of moves:

- Any action of the environment could be observed at any time between the moment when it becomes possible and the moment when it actually occurs.

- Dually, any action of the program could be observed at any time between the moment when it actually occurs and the moment it ceases to be possible.

To formalize this in terms of moves and plays, we define a preorder $\preceq$ on $P_{A}$ for any arena $A$ as the least transitive relation satisfying $s^{\prime} \preceq s$ for all $s, s^{\prime} \in P_{A}$ such that

1. $s^{\prime}=s_{0} \cdot o \cdot s_{1} \cdot s_{2}$ and $s=s_{0} \cdot s_{1} \cdot o \cdot s_{2}$, or

2. $s^{\prime}=s_{0} \cdot s_{1} \cdot p \cdot s_{2}$ and $s=s_{0} \cdot p \cdot s_{1} \cdot s_{2}$,

where $o$ is any $\mathrm{O}$ move and $p$ is any $\mathrm{P}$ move and every move in $s$ has the same justifier as in $s^{\prime}$. Since $s, s^{\prime}$ are legal plays by definition, it follows that no move in $s_{1}$ is justified by $o(1)$ and $p$ justifies no move in $s_{1}(2)$.

Definition 4. A strategy $\sigma$ is saturated if and only if whenever $s \in \sigma$ and $s^{\prime} \preceq s$ then $s^{\prime} \in \sigma$. 
The two saturation conditions, in various formulations, have a long pedigree in the semantics of concurrency. For example, they have been used by Udding to describe propagation of signals across wires in delay-insensitive circuits [10 and by Josephs et al to specify the relationship between input and output in asynchronous systems with channels [11. Laird has been the first to use them in game semantics, in his model of Idealized CSP [7].

For technical arguments it is convenient to use an equivalent "small-step" characterization of saturated strategies.

Lemma 2. $\sigma: A$ is saturated if and only if the two conditions below hold.
1. If $s m_{1} m_{2} \in \sigma$ and $\lambda_{A}\left(m_{1}\right)=\lambda_{A}\left(m_{2}\right)$ then $s m_{2} m_{1} \in \sigma$.
2. If spo $\in \sigma$ and sop $\in P_{A}$ then sop $\in \sigma$.

Recall that in the second clause it is necessary to stipulate $s o p \in P_{A}$ (Lemma 1).

Arenas and saturated strategies form a category $\mathcal{G}_{\text {sat }}$ in which $\mathcal{G}_{\text {sat }}(A, B)$ consists of saturated strategies on $A \Rightarrow B$. The identity strategy will be defined by saturating the strictly alternating copy-cat strategy, which is turn defined in the same way as identity strategies used for modeling sequential languages (but with respect to the new notion of positions).

Let $P_{A}^{\text {alt }}$ be the subset of $P_{A}$ consisting of alternating plays (no two consecutive moves are by the same player). The "alternating copy-cat strategy" $i d_{A}^{\text {alt }}$ is the least strategy containing $\left\{s \in P_{A_{1} \Rightarrow A_{2}}^{\text {alt }} \mid \forall t \sqsubseteq\right.$ even $\left.s, t \uparrow A_{1}=t \uparrow A_{2}\right\}$. In $i d_{A}^{\text {alt }} \mathrm{P}$ copies $\mathrm{O}$-moves as they come provided he is "fast enough" to do so before the next O-move; otherwise the strategy breaks down.

Recall the lack of switching conditions for $A_{1} \Rightarrow A_{2}$. Consequently, $i d_{A}^{\text {alt }}$ also admits plays of the shape $d_{2} d_{1} e_{1} e_{2} f_{1} f_{2}$, which are illegal in the alternating setting. We used subscripts 1,2 to indicate which instance of a type provides a move.

The identity strategy $i d_{A}$ will allow $\mathrm{P}$ to copy O-moves from one copy of $A$ to the other in a "parallel" fashion: the P-copy of an O-move does not have to follow the O-move immediately and can be delayed by some other O- or P-moves.

Definition 5. Let $\operatorname{sat}(\tau)$ be the least saturated strategy containing the strategy $\tau$. We define the identity strategy $i d_{A}$ as sat $\left(i d_{A}^{\text {alt }}\right)$.

The product and arena constructions make $\mathcal{G}_{\text {sat }}$ into a Cartesian closed category. The empty arena is the terminal object, pairing amounts to taking the sum (up to the canonical embeddings in the disjoint sum). Because the arenas $A \times B \Rightarrow C$ and $A \Rightarrow(B \Rightarrow C)$ are almost identical (up to associativity of disjoint sum), currying and un-currying essentially leave the strategies unchanged.

Proposition 1. $\mathcal{G}_{\text {sat }}$ is Cartesian closed.

The set of strategies on a given arena $A$ can be ordered by inclusion, which makes it into a complete lattice. The largest element $\top_{A}$ is $P_{A}$, the empty strategy $\perp_{A}$, in which positions are merely the initial O-moves, is the least element. Greatest lower bounds and lowest upper bounds are calculated by taking intersections and sums respectively. Saturated strategies inherit this structure because sums and intersections of saturated strategies remain saturated. 
Theorem 1. $\mathcal{G}_{\text {sat }}$ is an $\omega C P O$-enriched Cartesian closed category.

We finish with a technical lemma which shows that in some cases saturation is preserved by composition even though one of the strategies may not be saturated.

Lemma 3. If $\sigma: A \Rightarrow B, \tau: B \Rightarrow C$ are strategies, $\sigma$ is saturated and $C$ is flat then $\sigma ; \tau=\sigma ; \operatorname{sat}(\tau)$. In particular, $\sigma ; \tau: A \Rightarrow C$ is saturated.

As we shall see later, sometimes it will be convenient to use $\tau$ instead of $\operatorname{sat}(\tau)$ to simplify reasoning about composite strategies.

\subsection{The Game Model}

The lambda-calculus fragment of our language with fixed points can be modelled in a canonical way using the structure of $\mathcal{G}_{\text {sat }}$ exhibited in the previous section. In particular $\llbracket \operatorname{fix}\left(\lambda x^{\theta} \cdot x\right) \rrbracket=\perp \llbracket \theta \rrbracket$. We shall write $\Omega_{\theta}$ for $\operatorname{fix}\left(\lambda x^{\theta} . x\right)$.

Next we show how to interpret the other constructs. It is convenient to present an alternative, but equivalent syntax of the language using functions rather than term-forming combinators:

conditional : ifzero $_{\beta}: \exp \rightarrow \beta \rightarrow \beta \rightarrow \beta$

semaphores : grb : sem $\rightarrow$ com, rls : sem $\rightarrow$ com

commands : seq : com $\rightarrow \beta \rightarrow \beta$, parc : $\mathbf{c o m} \rightarrow \mathbf{c o m} \rightarrow \mathbf{c o m}$.

variables : assg : var $\rightarrow \exp \rightarrow$ com, deref : var $\rightarrow \exp$

arithmetic, logic : op : $\exp \rightarrow \exp \rightarrow \exp$.

binders : newvar $_{\beta}:(\operatorname{var} \rightarrow \beta) \rightarrow \beta$, newsem $_{\beta}:(\operatorname{sem} \rightarrow \beta) \rightarrow \beta$.

The strategies interpreting the functional constants of the language can be defined by giving the set of their complete plays.

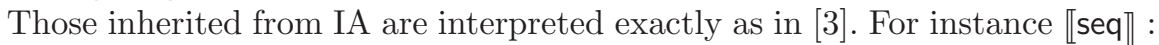
$\llbracket \mathbf{c o m} \rrbracket \Rightarrow \llbracket \beta \rrbracket_{0} \Rightarrow \llbracket \beta \rrbracket_{1}$ is given by positions of the shape $q_{1} \cdot r u n \cdot o k \cdot q_{0} \cdot a_{0} \cdot a_{1}$ and $\llbracket$ assg $\rrbracket: \llbracket \operatorname{var} \rrbracket_{0} \Rightarrow \llbracket \exp \rrbracket_{1} \Rightarrow \llbracket \operatorname{com} \rrbracket_{2}$ is defined by $\operatorname{run}_{2} \cdot q_{1} \cdot n_{1} \cdot$ write $(n)_{0} \cdot o k_{0} \cdot o k_{2}$.

The interpretations for $\llbracket \mathrm{grb} \rrbracket, \llbracket \mathrm{rls} \rrbracket: \llbracket \mathbf{s e m} \rrbracket_{0} \Rightarrow \llbracket \mathbf{c o m} \rrbracket_{1}$ are given respectively by the positions run $_{1} \cdot g r a b_{0} \cdot o k_{0} \cdot o k_{1}$ and run $_{1} \cdot$ release $_{0} \cdot o k_{0} \cdot o k_{1}$.

For parallel composition, $\llbracket$ parc $\rrbracket: \llbracket \mathbf{c o m} \rrbracket_{0} \Rightarrow \llbracket \mathbf{c o m} \rrbracket_{1} \Rightarrow \llbracket \mathbf{c o m} \rrbracket_{2}$ is the saturated strategy generated by $\operatorname{run}_{2} \cdot \operatorname{run}_{0} \cdot \operatorname{run}_{1} \cdot o k_{0} \cdot o k_{1} \cdot o k_{2}$. Thus, its complete plays are exactly those of $\operatorname{run}_{2} \cdot\left(r u n_{0} \cdot o k_{0} \amalg r u n_{1} \cdot o k_{1}\right) \cdot o k_{2}$. Note that this is the only language constant interpreted by a strategy with non-alternating plays.

\subsection{Stateful Behaviour: Cells and Locks}

The interpretation of a local variable is defined as the composition of the following strategies:

$$
\llbracket \Gamma \vdash \operatorname{newvar} x:=n \text { in } M: \beta \rrbracket=\Lambda_{x}(\llbracket \Gamma, x: \operatorname{var} \vdash M: \beta \rrbracket) ; \operatorname{cell}_{n}^{\beta}
$$

where $\Lambda_{x}$ is the currying isomorphism and the strategy cell ${ }_{n}^{\beta}:(\llbracket \operatorname{var} \rrbracket \Rightarrow \llbracket \beta \rrbracket) \Rightarrow$ $\llbracket \beta \rrbracket$ is the least strategy containing the alternating plays of the shape: $q \cdot q \cdot \mathrm{read}$. 
$n \cdot$ write $(i) \cdot o k \cdot \operatorname{read} \cdot i \cdots a \cdot a$, where $\mathrm{P}$ responds to each write $(i)$ with $o k$ and plays the most recently written value in response to read (or $n$ if no write $(i)$ has been played by $\mathrm{O}$ yet).

Local semaphore introduction is defined similarly:

$$
\llbracket \Gamma \vdash \operatorname{newsem} x:=n \text { in } M: \beta \rrbracket=\Lambda_{x}(\llbracket \Gamma, x: \operatorname{sem} \vdash M: \beta \rrbracket) ; \text { lock }_{n}^{\beta},
$$

where $\operatorname{lock}_{n}^{\beta}:(\llbracket \mathbf{s e m} \rrbracket \Rightarrow \llbracket \beta \rrbracket) \Rightarrow \llbracket \beta \rrbracket$ is the least strategy containing plays of the shape $q \cdot q \cdot \square \cdot a \cdot a$ where $\square$ is a segment of alternating grab $\cdot$ ok and release $\cdot o k$ sequences.

Equivalently, by Lemma 3, instead of cell ${ }_{n}^{\beta}$ and lock $_{n}^{\beta}$ one can use the saturated strategies sat $\left(\operatorname{cell}_{n}^{\beta}\right)$ and $\operatorname{sat}\left(\operatorname{lock}_{n}^{\beta}\right)$. Therefore, the above definitions always lead to saturated strategies, i.e. morphisms of $\mathcal{G}_{\text {sat }}$.

\subsection{Examples}

Example 1 (Nondeterminism). Let $M_{0}, M_{1}: \beta$. Define $M_{0}$ or $M_{1}: \beta$ as

$$
\text { newvar } x:=0 \text { in }\left((x:=0 \| x:=1) \text {; ifzero } ! x \text { then } M_{0} \text { else } M_{1}\right) .
$$

This construction can be extended to var, sem using mkvar, mksem respectively, and to higher-order types using $\eta$-expansion. Then we have $\llbracket M_{0}$ or $M_{1} \rrbracket=$ $\llbracket M_{0} \rrbracket \cup \llbracket M_{1} \rrbracket$.

Example 2 (Test of linearity). Consider a term $\Gamma \vdash M: \beta$ and an identifier $s: \mathbf{s e m}$. If $s$ is initialized to 0 and not used elsewhere, then $\operatorname{grab}(s) ; M$ behaves exactly like $M$, but can be used at most once if passed as argument to a function, as in $p: \beta \rightarrow \beta^{\prime} \vdash$ newsem $s:=0$ in $p(\operatorname{grab}(s) ; M)$. Observe that instantiating $p$ to $\lambda c: \operatorname{com} . c ; c$ or $\lambda c: \mathbf{c o m} . c \| c$ will not lead to convergence and that the corresponding strategy has no complete plays.

This construction can be extended to other types as in the case of or (Example [1) and plays an important role in the definability argument.

Example 3 (Test of linear parallelism). The following term generates only nonalternating complete plays:

$$
\begin{aligned}
& p: \operatorname{com}_{1} \rightarrow \operatorname{com}_{2} \rightarrow \operatorname{com}_{3} \vdash \operatorname{newsem} s_{l}, s_{r}, s:=0 \text { in } \\
& \quad p\left(\operatorname{grab}\left(s_{l}\right) ; \operatorname{grab}(s) ; \operatorname{grab}(s)\right)\left(\operatorname{grab}\left(s_{r}\right) ; \operatorname{release}(s) ; \operatorname{release}(s)\right): \operatorname{com}_{4} .
\end{aligned}
$$

They are generated, using saturation, by: $\operatorname{run}_{4} \cdot r u n_{3} \cdot r u n_{2} \cdot r u n_{1} \cdot o k_{2} \cdot o k_{1} \cdot o k_{3} \cdot o k_{4}$ in $\left(\llbracket \mathbf{c o m} \rrbracket_{1} \Rightarrow \llbracket \mathbf{c o m} \rrbracket_{2} \Rightarrow \llbracket \mathbf{c o m} \rrbracket_{3}\right) \rightarrow \llbracket \operatorname{com} \rrbracket_{4}$. Observe that instantiating $p$ to $\lambda c_{1}$ : com, $c_{2}$ : com. $c_{1} ; c_{2}$ leads to divergence and the corresponding strategy has no complete plays. However, we have convergence for $\lambda c_{1}: \operatorname{com}, c_{2}: \operatorname{com} . c_{1} \| c_{2}$.

For many programming tasks it is well known that semaphores can be programmed using shared variables only (e.g. the tie-breaker algorithms from [12]). However, such implementations have been defined with the assumption that the processes involved are distinct and can run different code. This does not seem uniform enough to program the behaviour required in Examples 2 and [3, where the competing threads are produced by the same piece of code. This apparent expressivity failure has motivated the introduction of semaphores as a primitive in our language. 


\section{Soundness and Adequacy}

Although $\mathcal{G}_{\text {sat }}$ can be shown to be inequationally sound, it is not fully abstract. As is the case for all game models, full abstraction will be proved for the quotient of $\mathcal{G}_{\text {sat }}$ with respect to the so-called intrinsic preorder. Fortunately, in our case the quotient turns out to have a more explicit representation based on complete plays (like for IA, but not PCF), which makes it easy to apply our model to reasoning about program approximation and equivalence.

Let $\Sigma$ be the game with a single question $q$ and one answer $a$ such that $q \vdash_{\Sigma} a$ (note that $\Sigma$ is essentially the same as $\llbracket \mathbf{c o m} \rrbracket$ ). There are two strategies for $\Sigma$ : the bottom strategy $\perp_{\Sigma}$ and the top strategy $\top_{\Sigma}=\{\epsilon, q, q \cdot a\}$. The intrinsic preorder for saturated strategies on $A$ is defined by $\tau_{1} \lesssim \tau_{2}$ iff $\forall \alpha \in$ $\mathcal{G}_{\text {sat }}(A, \Sigma)$ if $\tau_{1} ; \alpha=\top_{\Sigma}$ then $\tau_{2} ; \alpha=\top_{\Sigma}$. For composition the strategies $\tau_{i}: A$ are regarded as ones between 1 and $A$.

Theorem 2 (Characterization). Let $\tau_{1}, \tau_{2}$ be saturated strategies on $A . \tau_{1} \lesssim$ $\tau_{2}$ if and only if $\operatorname{comp}\left(\tau_{1}\right) \subseteq \operatorname{comp}\left(\tau_{2}\right)$.

Because the quotient $\mathcal{G}_{\text {qsat }}=\mathcal{G}_{\text {sat }} / \lesssim$ has such a direct representation based on inclusion of complete plays, it is easy to see that it is also a $\omega$ CPO-enriched category. The compact elements of $\mathcal{G}_{\text {qsat }}$ are precisely the equivalence classes $[\sigma]_{\lesssim} \operatorname{such}$ that $\operatorname{comp}(\sigma)$ is finite. Next we examine the theoretical properties of $\mathcal{G}_{\text {qsat }}$ : soundness, adequacy and, finally, full-abstraction.

For the purpose of relating our model with the operational semantics we will represent a state $s: \Sigma \rightarrow \mathbb{N}$ by the strategy $\llbracket s \rrbracket_{\beta}:\left(\llbracket \theta_{1} \rrbracket \Rightarrow \cdots \Rightarrow \llbracket \theta_{m} \rrbracket \Rightarrow\right.$ $\llbracket \beta \rrbracket) \Rightarrow \llbracket \beta \rrbracket$ generated from complete plays of the shape $q \cdot q \cdot \square \cdot a \cdot a$ where $\square$ stands for a (possibly empty) sequence of segments of one of the following shapes: read $\cdot n$, write $(n) \cdot o k, g r a b \cdot o k$ or release $\cdot o k$ such that the projections onto $\theta_{i}$ are of the same shape as those of suitably initialized (i.e. $n_{i}=s\left(l_{i}\right)$ ) cell ${ }_{n_{i}}^{\beta}$ or lock $_{n_{i}}^{\beta}$ strategies. We can think of $\llbracket s \rrbracket_{\beta}$ as a "super-sequentialized" store, where individual cells and locks are accessed sequentially both individually and as a group. In what follows, $\llbracket \Sigma \vdash M: \beta \rrbracket ; \llbracket s \rrbracket_{\beta}$ will be the interpretation of $\Sigma \vdash M: \beta$ at state $s$. Recall that, by Lemma 3 , the same result would be achieved by using $\operatorname{sat}\left(\llbracket s \rrbracket_{\beta}\right)$.

Lemma 4. For any term $\Sigma \vdash M: \beta$ and any any state $s$, if $\Sigma \vdash M, s \longrightarrow M^{\prime}, s^{\prime}$ then $\llbracket \lambda \boldsymbol{x} . M^{\prime} \rrbracket ; \llbracket s^{\prime} \rrbracket_{\beta} \subseteq \llbracket \lambda \boldsymbol{x} . M \rrbracket ; \llbracket s \rrbracket_{\beta}$.

Soundness then follows.

Proposition 2 (Soundness). For any $\Sigma \vdash M: \beta$ and any state $s$, if $\Sigma \vdash$ $M, s \Downarrow$ then $\llbracket \lambda \boldsymbol{x} . M \rrbracket ; \llbracket s \rrbracket_{\beta} \neq \perp$.

Our semantic model is adequate in the usual sense. The proof uses logical relations, adapted to small-step operational semantics.

Proposition 3 (Computational adequacy). For any program $P, P \Downarrow$ if and only if $\llbracket P \rrbracket \neq \perp$. 


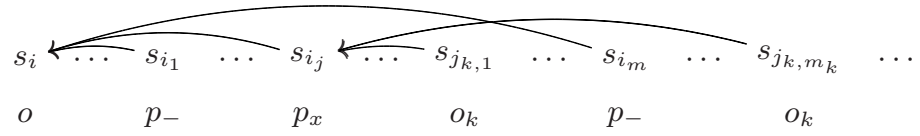

Fig. 1. Questions and justification pointers

Together, the two propositions imply:

Theorem 3 (Inequational Soundness). Let $\Gamma \vdash M_{i}: \theta$ for $i=1,2$. If $\llbracket M_{1} \rrbracket \lesssim \llbracket M_{2} \rrbracket$ then $M_{1} \check{\sim}_{\theta} M_{2}$. Equivalently, comp $\left(\llbracket M_{1} \rrbracket\right) \subseteq \operatorname{comp}\left(\llbracket M_{2} \rrbracket\right)$ implies $M_{1} \check{\sigma}_{\theta} M_{2}$.

\section{$5 \quad$ Full Abstraction}

We give a direct recursive algorithm, called $P R O C^{+}$, which, given a position $s$ of $\llbracket \theta \rrbracket$, returns a term of type $\theta$ whose denotation is the smallest saturated strategy containing $s$.

The basic idea of the construction is to use justification pointers to identify potential threads. If two moves are justified by the same move we can think of them as occurring in parallel threads spawned by the thread corresponding to the justifier. When constructing the term for the position we compose all these threads in parallel. Then we use specially designated side-effects as time-stamps to enforce the particular order of moves that happens in the position. Of course, we can only try to achieve this up to the saturation conditions.

In order to generate the desired positions we need to control the way in which both $\mathrm{P}$ and $\mathrm{O}$ move. We control $\mathrm{P}$-moves using guards that wait for special sideeffects (time-stamps) caused by O-moves. The effects take place only if a correct O-move is played and we make sure that they occur only once by using a fresh semaphore for each O-move. This allows us to enforce arbitrary synchronization policies, restricting the order of moves present in the original sequence up to the reorderings dictated by the saturation conditions. Each O-move $s_{j}$ produces an associated time-stamp which is stored in a variable $x_{j}$, bound by new at the top level and initialized to 0. We "time-stamp" the variable by assigning 1 to it. For $1 \leq j \leq|s|-1$, let $O_{j}=\left\{i \in \mathbb{N} \mid 0 \leq i<j, s_{i}\right.$ is an O-move $\}$. Let test $\equiv$


checks for time-stamps originating from all the O-moves with indices smaller than $j: W A I T_{j} \equiv \operatorname{test}\left(1-! x_{g_{1}}\right) ; \cdots ; \operatorname{test}\left(1-! x_{g_{k}}\right)$, with $O_{j}=\left\{g_{1}, \ldots, g_{k}\right\}$.

Below we give the definition of $\operatorname{PROC}^{+}(s: \theta)$ for the case where $\theta$ is generated from com only (see also the example). The complete definition is available in 13 .

$P R O C^{+}$first calls $P R O C$ and then adds bindings to the term returned by $P R O C$. The initial argument to $P R O C$ is the original position $s$. In the recursive invocations, the argument is a subsequence of the form $s \uparrow m$, where $t \uparrow m$ is the 
subsequence of $t$ consisting of $m$ and all moves hereditarily justified by $m$, always an O-question. Note that consequently a move in $t$ is answered in $t$ if and only if it is answered in $s$. PROC uses indices relative to the original $s$; we write $s_{i}$ for the $i$ th move of $s$, assuming $s_{0}$ initial. $\operatorname{PROC}(t: \theta)$ where $\theta=\theta_{1} \rightarrow \ldots \rightarrow \theta_{h} \rightarrow$ com is defined in two stages which manage $\mathrm{O}$-questions and $\mathrm{P}$-answers, and respectively $\mathrm{P}$-questions and $\mathrm{O}$-answers. If $t$ is empty, $\lambda p_{1} \cdots p_{h} . \Omega_{\theta_{0}}$ is returned. Otherwise, let $o=s_{i}$ be the initial move of $t$ (which is always an O-question).

1. Let $p_{1}, \cdots, p_{h}$ be all the P-questions enabled by $o$ (corresponding respectively to $\left.\theta_{1}, \cdots, \theta_{h}\right)$. Let $i_{1}<\cdots<i_{m}$ be the $s$-indices of all occurrences of $p_{1}, \cdots, p_{h}$ in $t$ which are explicitly justified by $s_{i}$ (see Figure1). Then PROC returns $\lambda p_{1} \cdots p_{h} .\left(x_{i}:=1\right) ;\left(P_{1}\|\cdots\| P_{m}\right) ; P A N S_{i}^{\text {com }}$ where $P_{1}, \cdots, P_{m}$ are defined in 2. and $P A N S_{i}^{\text {com }}$ is either $\Omega_{\text {com }}$ (if $s_{i}$ is unanswered in $t$ ) or $W_{A I T} i_{i^{\prime}}\left(\right.$ if $s_{i^{\prime}}$ answers $s_{i}$ in $t$ ). By convention, $\left(P_{1}\|\cdots\| P_{m}\right)$ degenerates to skip for $m=0$.

2. Here we show how to define he terms $P_{j}$ for $1 \leq j \leq m$. Let us fix $j$ and suppose that $s_{i_{j}}=p_{x}(1 \leq x \leq h)$ and $\theta_{x}=\theta_{1}^{\prime} \rightarrow \ldots \rightarrow \theta_{n}^{\prime} \rightarrow \mathbf{c o m}$. Let $o_{1}, \cdots, o_{n}$ be all the O-questions enabled by $p_{x}$ (corresponding to $\theta_{1}^{\prime}, \cdots, \theta_{n}^{\prime}$ respectively).

For each $k(1 \leq k \leq n)$ let $j_{k, 1}<\cdots<j_{k, m_{k}}$ be the $s$-indices of all occurrences of $o_{k}$ in $t$ which are explicitly justified by $s_{i_{j}}$ (see Figure 1).

If $m_{k}=0$, then $P_{j}^{k} \equiv \Omega_{\theta_{k}^{\prime}}$. Otherwise, for all $l=1, \cdots, m_{k}$ we make the following definitions: $P_{j}^{k, l} \equiv \operatorname{PROC}\left(t \uparrow s_{j_{k, l}}: \theta_{k}^{\prime}\right)$ and

$$
P_{j}^{k} \equiv O N C E_{w_{j_{k, 1}}}\left[P_{j}^{k, 1}\right] \text { or } \cdots \text { or } O N C E_{w_{j_{k, m_{k}}}}\left[P_{j}^{k, m_{k}}\right]
$$

where $w_{j_{k, 1}}, \ldots, w_{j_{k, m}}$ are fresh semaphore names. The construction or is defined as in Example[1, and $O N C E_{w}[M]=\operatorname{grab}(w) ; M$ as in Example 2 $P_{j} \equiv W A I T_{i_{j}} ;\left(p_{x} P_{j}^{1} \cdots P_{j}^{n}\right) ; O A N S_{i_{j}}^{\text {com }}$ where $O A N S_{c}^{\text {com }}$ is skip (if $s_{c}$ is unanswered in $t$ ) or $x_{c^{\prime}}:=1$ (when $s_{c^{\prime}}$ answers $s_{c}$ in $t$ ).

After $\operatorname{PROC}(s: \theta)$ returns $\lambda p_{1} \cdots p_{k} \cdot M$, all variables and semaphores $\left(x_{-}, w_{-}\right)$ used in the construction of $M$ must be bound at the topmost level (the variables $x_{-}$must be initialized to 0 , the semaphores $w_{-}$to 0 ) by taking

$$
\lambda p_{1} \cdots p_{k} \cdot \text { newvar } \boldsymbol{x}:=\mathbf{0} \text { in }(\text { newsem } \boldsymbol{w}:=\mathbf{0} \text { in } M)
$$

We denote the final term by $\operatorname{PROC}^{+}(s: \theta)$.

Example 4. Consider the play

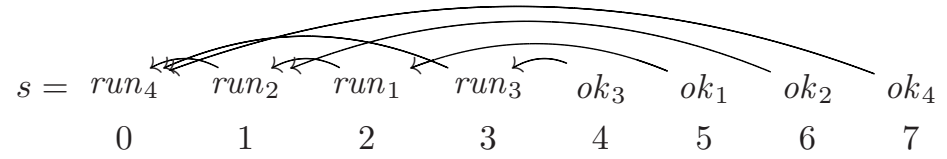


in arena $\llbracket\left(\mathbf{c o m}_{1} \rightarrow \mathbf{c o m}_{2}\right) \rightarrow \mathbf{c o m}_{3} \rightarrow \mathbf{c o m}_{4} \rrbracket$. The term $\lambda f . \lambda x . f x$ has this play among its complete positions. The term $\operatorname{PROC}^{+}(s)$ is:

$$
\begin{aligned}
& \lambda f . \lambda a . \text { newvar } x_{0}, x_{2}, x_{4}, x_{6}:=0 \text { in newsem } w_{2}:=0 \text { in } \\
& x_{0}:=1 \\
& \left(\left(W_{A} T_{1} ; f\left(O N C E_{w_{2}}\left[x_{2}:=1 ; W A I T_{5}\right]\right) ; x_{6}:=1\right) \|\left(W A I T_{3} ; a ; x_{4}:=1\right)\right) ; \\
& \mathrm{WAIT}_{7}
\end{aligned}
$$

Notice that the second argument $a$ can be evaluated only after the first one $(f)$ is, because of $W A I T_{3}$. On the other hand, a must be evaluated before $f$ 's argument because of $W A I T_{5}$. The resulting temporal ordering of the moves is, consequently, the same as in $f(a)$.

Using $\mathrm{PROC}^{+}$and or we can show the following result:

Theorem 4 (Compact Definability). Any compact saturated strategy $\sigma$, i.e. one generated by a finite set of positions, is definable.

With adequacy and definability established, full abstraction follows routinely.

Theorem 5 (Full abstraction). Let $\Gamma \vdash M, N: \theta$. Then $\llbracket M \rrbracket \lesssim \llbracket N \rrbracket$ if and only if $M \check{\sim}_{\theta} N$. Equivalently, by the Characterization Theorem (Thm. (2), $\operatorname{comp}(\llbracket M \rrbracket) \subseteq \operatorname{comp}(\llbracket N \rrbracket)$ if and only if $M \check{\sim}_{\theta} N$.

\section{Conclusion}

We have presented a fully abstract game model for a programming language with fine-grained shared-variable concurrency. We found that HO-style games are naturally suited to interpreting concurrency, and most of the technical complexity required for modeling sequential computation can be avoided. Therefore, we can give a direct definability construction, as opposed to the usual factorization method.

In addition to its theoretical interest, our fully abstract model can be used to reason about program may-equivalence. We can make straightforward arguments about ground-type equivalences such as Brookes's laws of parallel programming [1], or other typical second-order equivalences. In order for such arguments to be formalized, and even automated, it is necessary to find a concrete representation of strategies, along the lines of [14]. For this purpose, the most convenient representations are those which are finite-state, such as regular expressions, regular languages, labelled transitions systems, etc. Such a representation can be easily integrated in our ongoing research effort in game-based software model checking 15]. However, identifying a non-trivial fragment of this language for which the strategies are finitary is not straightforward.

The main theoretical development which is required is adapting our model to dealing with must-equivalence, i.e. a notion of equivalence which considers not just termination but the full spectrum of observable behaviour: termination, failure and divergence. Must-equivalence has been studied using game semantics in the simpler setting of bounded nondeterminism by Harmer and McCusker [16], and some of their techniques may be applicable in our setting. 
Acknowledgements. We are grateful to Samson Abramsky and Guy McCusker for stimulating discussions on the subject matter of the paper.

\section{References}

1. Brookes, S.: The essence of Parallel Algol. Chapter 21 of [2].

2. O'Hearn, P.W., Tennent, R.D., eds.: Algol-like Languages. Progress in Theoretical Computer Science. Birkhäuser (1997)

3. Abramsky, S., McCusker, G.: Linearity, sharing and state: a fully abstract game semantics for Idealized Algol with active expressions. Chapter 20 of [2].

4. Hyland, J. M. E., Ong, C.-H. L.: On full abstraction for PCF: I, II and III. Information and Computation 163(2) (2000) 285-408

5. Abramsky, S., Melliès, P. A.: Concurrent games and full completeness. In: Proceedings of LICS (1999) 431-442

6. Abramsky, S.: Game semantics of Idealized Parallel Algol. Lecture given at the Newton Institute (1995)

7. Laird, J.: A games semantics of Idealized CSP. In: Volume 45 of Electronic Notes in Theoretical Computer Science (2001) 157-176

8. Brookes, S.: Full abstraction for a shared variable parallel language. Chapter 21 of $[2$.

9. Röckl, C., Sangiorgi, D.: A $\pi$-calculus process semantics of Concurrent Idealized Algol. In: Proceedings of FOSSACS, Volume 1578 of LNCS (1999) 306-322

10. Udding, J.T.: A formal model for defining and classifying delay-insensitive circuits and systems. Distributed Computing 1(4) (1986) 197-204

11. Jifeng, H., Josephs, M.B., Hoare, C.A.R.: A theory of synchrony and asynchrony. In: Programming Concepts and Methods. Elsevier (1990) 459-473

12. Andrews, G.: Concurrent Programming: principles and practice. Addison-Wesley Publishing Company (1991)

13. Ghica, D. R., Murawski, A. S.: Angelic semantics of fine-grained concurrency. Technical Report PRG-RR-03-20. Oxford University Computing Laboratory (2003)

14. Ghica, D. R., McCusker, G.: Reasoning about Idealized ALGOL using regular languages. In: Proceedings of ICALP. Volume 1853 of LNCS (2000) 103-116

15. Abramsky, S., Ghica, D. R., Murawski, A. S., Ong, C.-H. L.: Algorithmic game semantics and component-based verification. In: Proceedings of TACAS. LNCS (2004)

16. Harmer, R., McCusker, G.: A fully abstract game semantics for finite nondeterminism. In: Proceedings of LICS (1999) 422-430 